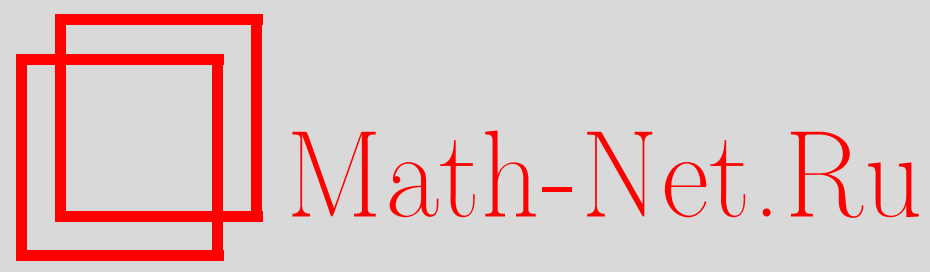

В. М. Евтухов, Об условиях неколеблемости решений одного нелинейного дифференциального уравнения второго порядка, Матем. заметки, 2000, том 67, выпуск 2, 201-210

DOI: https://doi.org/10.4213/mzm828

Использование Общероссийского математического портала Math-Net.Ru подразумевает, что вы прочитали и согласны с пользовательским соглашением http://www.mathnet.ru/rus/agreement

Параметры загрузки:

IP : 54.224 .187 .69

26 апреля 2023 г., 13:03:31

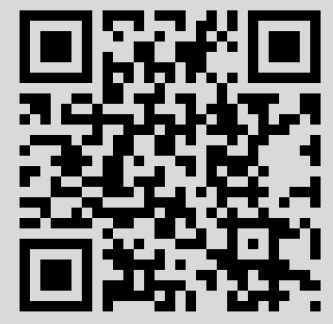




\title{
ОБ УСЛОВИЯХ НЕКОЛЕБЛЕМОСТИ РЕШЕНИЙ ОДНОГО НЕЛИНЕЙНОГО ДИФФЕРЕНЦИАЛЬНОГО УРАВНЕНИЯ ВТОРОГО ПОРЯДКА
}

\author{
В. М. Евтухов
}

Для нелинейного дифференциального уравнения

$$
y^{\prime \prime}+p(t)|y|^{\sigma}\left|y^{\prime}\right|^{\lambda} \operatorname{sign} y=0,
$$

где $\sigma>-1, \lambda<1$ и $p:[a,+\infty[\rightarrow] 0,+\infty[-$ непрерывная функция, установлены достаточные признаки неколеблемости всех правильных решений, которые в случае классического обобщенного уравнения Эмдена-Фаулера $(\lambda=0)$ дополняют известные результаты.

Библиографоия: 12 названий.

1. Формулировка основных теорем. Рассмотрим дифференциальное уравнение

$$
y^{\prime \prime}+p(t)|y|^{\sigma}\left|y^{\prime}\right|^{\lambda} \operatorname{sign} y=0,
$$

где $\sigma>-1, \lambda<1$ и $p:[a,+\infty[\rightarrow] 0,+\infty[-$ непрерьвная функция.

Под решением этого уравнения будем понимать любую непрерьвно дифференцируемую на некотором связном промежутке $I \subset[a,+\infty[$ функцию, которая удовлетворяет (1.1) всюду на $I$ за возможным исключением (если соблюдается хотя бы одно из неравенств $\sigma \leqslant 0$ или $\lambda<0)$ не более чем счетного множества нулей $y$ и $y^{\prime}$.

Решение $y$ уравнения (1.1) назовем правильным, если оно определено на промежутке $\left[t_{0},+\infty\left[\subset\left[a,+\infty\left[\right.\right.\right.\right.$, а его производная $y^{\prime}$ на любом замкнутом отрезке $\left[\tau_{1}, \tau_{2}\right]$ этого промежутка не более конечного числа раз обращается в нуль.

Из условия $p(t)>0$ при $t \geqslant a$ непосредственно следует, что для каждого правильного решения $y:\left[t_{0},+\infty[\rightarrow \mathbb{R}\right.$ уравнения $(1.1)$ соблюдается неравенство

$$
|y(t)|+\left|y^{\prime}(t)\right|>0 \text { при } t \geqslant t_{0} .
$$

Правильное решение назовем колеблющимся, если оно имеет последовательность нулей, сходящуюся к $+\infty$, и неколеблющимся-в противном случае.

Эта работа была частично поддержана грантом «APU061032» Международной Соросовской Программы поддержки образования в области точных наук в Украине. 
Нетрудно заметить, что указанные выше условия $\sigma>-1, \lambda<1$ и $p(t)>0$ являются в случае знакоопределенной функции $p$ необходимыми для существования у уравнения (1.1) правильных колеблюшихся решений.

При $\lambda=0$ уравнение (1.1) имеет вид

$$
y^{\prime \prime}+p(t)|y|^{\sigma} \operatorname{sign} y=0
$$

и носит название обобщенного уравнения Эмдена-Фаулера.

Условия неколеблемости всех правильных решений уравнения (1.2) при $0<\sigma \neq 1$ устанавливались в работах И.Т. Кигурадзе [1], 3. Нехари [2], Киу-Лянг Чиу [3], [4], Дж. С.В. Уонга [5] и других авторов (см. монографию И. Т. Кигурадзе, Т. А. Чантурия $[6, \S 18$, с. $344-347]$ и обзорные статьи [7], [8]).

Для уравнения (1.1) при $\sigma+\lambda \neq 1$ известен лиш аналог результата И. Т. Кигурадзе [1], вытекающий из теоремы 1 работы Ч. А. Схаляхо [9] и соответствующий случаю $\sigma+\lambda>1$. Случай $\sigma+\lambda=1$ исследовался в [10] и в работах Д. Д. Мирзова [11], [12].

В настоящей заметке устанавливаются следующие три признака неколеблемости всех правильных решений уравнения (1.1).

Теорема 1. Если $\sigma+\lambda<1, p(t)=p_{0}(t)-p_{1}(t), p_{0}, p_{1}:[a,+\infty[\rightarrow[0,+\infty[-$ локально абсолютно непрерывны, не убывают и

$$
\lim _{t \rightarrow+\infty} t^{2-\lambda} p(t) \exp \left[\frac{1-\sigma-\lambda}{\sigma+1} \int_{a}^{t} \frac{d p_{0}(t)}{p(t)}\right]=0,
$$

то каждое правильное решение уравнения (1.1) является неколеблющимся.

Теорема 2. Если $\sigma+\lambda>1, \quad p(t)=p_{0}(t)-p_{1}(t), p_{0}, p_{1}:[a,+\infty[\rightarrow[0,+\infty[-$ локально абсолютно непрерывны, не убывают и

$$
\lim _{t \rightarrow+\infty} t^{\sigma+1} p(t) \exp \left[\frac{\sigma+\lambda-1}{2-\lambda} \int_{a}^{t} \frac{d p_{0}(\tau)}{p(\tau)}\right]=0,
$$

то каждое правильное решение уравнения (1.1) является неколеблющимся.

ТЕорема 3. Пусть $\sigma+\lambda>1$ и для некоторого числа $\varepsilon>0$, удовлетворяющего неравенству

$$
\varepsilon \geqslant 1-\frac{\lambda(\sigma+1)}{2-\lambda}
$$

функиия

$$
\Phi(t)=p(t) t^{\frac{2-\lambda+(1-\lambda)(\sigma+1)}{2-\lambda}}(\ln t)^{\frac{\sigma+1}{2-\lambda}+\varepsilon}
$$

не возрастает при $t \geqslant b$, где $b>\max \{a, 1\}$. Тогда каждое правильное решение уравнения (1.1) является неколеблющимся.

ЗАМЕЧАНИЕ 1. Из теорем 1 и 2 при выполнении дополнительных условий

$$
\lambda=0, \quad \sigma>0, \quad \int_{a}^{+\infty} \frac{d p_{0}(\tau)}{p(\tau)}<+\infty
$$

получаем теоремы Уонга [5], установленные для уравнения (1.2). Предлагаемьй в п. 3 метод доказательства теорем 1 и 2 позволяет (в отличие от [5]) охватить при $\lambda=0$ и случай, когда $-1<\sigma \leqslant 0$. 
ЗАмЕчАниЕ 2. Теорема 3 при $\lambda=0$ и $\varepsilon=1$ совпадает с теоремой Нехари из [2].

\section{2. Вспомогательные предложения.}

Лемма 1. Пусть $p(t)=p_{0}(t)-p_{1}(t), p_{0}, p_{1}:[a,+\infty[\rightarrow[0,+\infty[-$ локально абсолютно непрерывны $и$ не убывают. Тогда любое непродолэсаемое решение $y$ уравнения (1.1) определено в промежутке $[a,+\infty[u$ при $t>s \geqslant a$ соблюдаются неравенства

$$
\begin{aligned}
& \rho_{1}(y)(s) \exp \left(-\int_{s}^{t} \frac{d p_{0}(\tau)}{p(\tau)}\right) \leqslant \rho_{1}(y)(t) \leqslant \rho_{1}(y)(s) \exp \left(\int_{s}^{t} \frac{d p_{1}(\tau)}{p(\tau)}\right), \\
& \rho_{2}(y)(s) \exp \left(-\int_{s}^{t} \frac{d p_{1}(\tau)}{p(\tau)}\right) \leqslant \rho_{2}(y)(t) \leqslant \rho_{2}(y)(s) \exp \left(\int_{s}^{t} \frac{d p_{0}(\tau)}{p(\tau)}\right),
\end{aligned}
$$

$2 \partial e$

$\rho_{1}(y)(t)=\frac{\left|y^{\prime}(t)\right|^{2-\lambda}}{p(t)}+\frac{2-\lambda}{\sigma+1}|y(t)|^{\sigma+1}, \quad \rho_{2}(y)(t)=\left|y^{\prime}(t)\right|^{2-\lambda}+\frac{2-\lambda}{\sigma+1} p(t)|y(t)|^{\sigma+1}$.

ДокАЗАТЕльСТво. Пусть $y:] t_{1}, t_{2}[\rightarrow \mathbb{R}-$ произвольное непродолжаемое решение уравнения (1.1). Покажем, что это решение при $t_{1}<s<t<t_{2}$ удовлетворяет неравенствам (2.1). Действительно, согласно условиям леммы и (1.1) функция $\rho_{1}(y)(t)$ абсолютно непрерьвна на $[s, t]$ и

$$
\left(\rho_{1}(y)(t)\right)^{\prime}=\frac{\left|y^{\prime}(t)\right|^{2-\lambda}}{p^{2}(t)}\left[p_{1}^{\prime}(t)-p_{0}^{\prime}(t)\right]
$$

почти всюду на $] t_{1}, t_{2}\left[\right.$. Поэтому для любого $\varepsilon>0$ при $t_{1}<s<t<t_{2}$ имеет место равенство

$$
\rho_{1}(y)(t)+\varepsilon=\left(\rho_{1}(y)(s)+\varepsilon\right) \exp \left(\int_{s}^{t} \frac{\left|y^{\prime}(\tau)\right|^{2-\lambda}}{\rho_{1}(y)(\tau)+\varepsilon} \frac{d p_{1}(\tau)}{p^{2}(\tau)}-\int_{s}^{t} \frac{\left|y^{\prime}(\tau)\right|^{2-\lambda}}{\rho_{1}(y)(\tau)+\varepsilon} \frac{d p_{0}(\tau)}{p^{2}(\tau)}\right)
$$

Отсюда, учитьвая, что $\left|y^{\prime}(\tau)\right|^{2-\lambda} \leqslant p(\tau) \rho_{1}(y)(\tau)$ при $\tau \in[s, t]$ и $p_{0}, p_{1}-$ не убьвают, в силу произвольности $\varepsilon>0$ получим (2.1). Установленные при $t_{1}<s<t<t_{2}$ неравенства (2.1), очевидно, не противоречат тому, что $y:] t_{1}, t_{2}[\rightarrow \mathbb{R}$ - непродолжаемое решение уравнения (1.1) лишь в случаях, когда $t_{1}=a, t_{2}=+\infty$ и $a$ входит в область определения $y$.

Неравенства (2.2) для каждого решения $y:[a,+\infty[\rightarrow \mathbb{R}$ уравнения (1.1) доказьваются таким же образом, как и неравенства (2.1), с использованием соотношения

$$
\begin{aligned}
\rho_{2}(y)(t)+\varepsilon= & \left(\rho_{2}(y)(s)+\varepsilon\right) \\
& \times \exp \left\{\frac{2-\lambda}{\sigma+1}\left(\int_{s}^{t} \frac{p(\tau)|y(\tau)|^{\sigma+1}}{\rho_{2}(y)(\tau)+\varepsilon} \frac{d p_{0}(\tau)}{p(\tau)}-\int_{s}^{t} \frac{p(\tau)|y(\tau)|^{\sigma+1}}{\rho_{2}(y)(\tau)+\varepsilon} \frac{d p_{1}(\tau)}{p(\tau)}\right)\right\},
\end{aligned}
$$

справедливого при $t>s \geqslant a$ и любом $\varepsilon$. Лемма доказана. 
ЗАмЕчАНИЕ 3. При выполнении условий леммы 1 начальным условиям $y\left(t_{0}\right)=$ $y^{\prime}\left(t_{0}\right)=0$, где $t_{0} \in[a,+\infty[$, может удовлетворять только тривиальное решение $(y(t) \equiv 0$ на промежутке $[a,+\infty[)$ уравнения (1.1), если оно существует.

Принимая во внимание это замечание, нетрудно установить

СлЕдСТВИЕ 1. Если соблюдаются условия леммы 1, то каждое нетривиальное решение уравнеия (1.1) на замкнутом промежутке $[a, b] \subset[a,+\infty[$ имеет не более конечного числа нулей.

ЗАмЕчАниЕ 4. Лемма 1 и следствие 1 , очевидно, справедливы и для уравнения

$$
y^{\prime \prime}\left|y^{\prime}\right|^{-\lambda}+p(t)|y|^{\sigma} \operatorname{sign} y=0
$$

полученного из (1.1) делением на $\left|y^{\prime}\right|^{\lambda}$. При этом нетрудно заметить, что каждое решение уравнения (2.3), отличное от тривиального (если оно сушествует), является правильньг.

Значит, для уравнения (1.1) имеет место

СлЕДСТВИЕ 2. Пусть соблюдаются условия леммы 1. Тогда: 1) при $\lambda<0, a$ также при $\lambda=0 u-1<\sigma \leqslant 0$ все решения уравнения (1.1) являются правильньми; 2) при $\lambda=0 и \sigma>0$ любое нетривиальное решение уравнения (1.1) является правильным; 3) при $0<\lambda<1$ кажсде решение уравнения (2.3) является правильным решением уравнения (1.1).

Лемма 2. Пусть $y:[a,+\infty[\rightarrow \mathbb{R}-$ правильное колеблющееся решение уравнения (1.1) и $\mathcal{T}_{y}$-множество его нулей и точек локального әкстремума. Тогда для любой непрерывно-дифферениируемой функиии $\varphi:\left[a,+\infty\left[\rightarrow \mathbb{R} u\right.\right.$ любых $\tau_{1}, \tau_{2} \in \mathcal{T}_{y}$

$$
\begin{aligned}
& \int_{\tau_{1}}^{\tau_{2}} \varphi(\tau)\left|y^{\prime}(\tau)\right|^{2-\lambda} d \tau=(1-\lambda) \int_{\tau_{1}}^{\tau_{2}} \varphi(\tau) p(\tau)|y(\tau)|^{\sigma+1} d \tau \\
&-\int_{\tau_{1}}^{\tau_{2}} \varphi^{\prime}(\tau)\left|y^{\prime}(\tau)\right|^{1-\lambda} y(\tau) \operatorname{sign} y^{\prime}(\tau) d \tau
\end{aligned}
$$

Доказательство этой леммы непосредственно вытекает из равенства

$$
\begin{gathered}
\int_{s}^{t} d\left(\varphi(\tau)\left|y^{\prime}(\tau)\right|^{1-\lambda} y(\tau) \operatorname{sign} y^{\prime}(\tau)\right)=\int_{s}^{t} \varphi^{\prime}(\tau)\left|y^{\prime}(\tau)\right|^{1-\lambda} y(\tau) \operatorname{sign} y^{\prime}(\tau) d \tau \\
+\int_{s}^{t} \varphi(\tau)\left[\left|y^{\prime}(\tau)\right|^{2-\lambda}-(1-\lambda) p(\tau)|y(\tau)|^{\sigma+1}\right] d \tau, \quad \text { где } t>s \geqslant a
\end{gathered}
$$

\section{3. Доказательства основных теорем.}

ДокАЗАТЕЛЬСтво тЕоремы 1. Допустим противное, т.е. что уравнение (1.1) имеет правильное колеблющееся решение $y$. Это решение согласно лемме 1 определено на промежутке $[a,+\infty[$ и при $t>s \geqslant a$ удовлетворяет неравенствам (2.1). Отсюда с учетом определения правильного решения следует, что

$$
\rho_{1}(y)(t)>0 \quad \text { при } \quad t \geqslant a .
$$


В дальнейшем решение $y$ будем исследовать на промежутке $\left[t_{0},+\infty\left[\right.\right.$, где $t_{0}=$ $\max \{1, a\}$.

Уравнение (1.1) с помощью замен переменных

$$
y(t)=t^{\frac{1-\lambda}{2-\lambda}} \xi(\tau), \quad \tau=\ln t
$$

сведем к уравнению

$$
\left(\xi^{\prime}+\frac{1-\lambda}{2-\lambda} \xi\right)^{\prime}-\frac{1}{2-\lambda}\left(\xi^{\prime}+\frac{1-\lambda}{2-\lambda} \xi\right)+p(t) t^{1+\gamma}|\xi|^{\sigma}\left|\xi^{\prime}+\frac{1-\lambda}{2-\lambda} \xi\right|^{\lambda} \operatorname{sign} \xi=0
$$

где $t=e^{\tau}$ и

$$
\gamma=\frac{(1-\lambda)(\sigma+1)}{2-\lambda}
$$

Указанному вьше решению $y:\left[t_{0},+\infty[\rightarrow \mathbb{R}\right.$ уравнения (1.1) в силу (3.2) и (3.1) соответствует колеблюшееся решение $\xi:\left[\tau_{0},+\infty\left[\rightarrow \mathbb{R}\left(\tau_{0}=\ln t_{0}\right)\right.\right.$ уравнения (3.3), удовлетворяющее неравенству

$$
|\xi(\tau)|+\left|\xi^{\prime}(\tau)\right|>0 \text { при } \tau \geqslant \tau_{0} .
$$

Поэтому существует последовательность $\left\{\tau_{k}\right\}$ точек локального максимума $\xi$, сходящаяся к $+\infty$ и такая, что $\xi\left(\tau_{k}\right)>0$ при $k=1,2, \ldots$. Для каждой точки $\tau_{k}$ этой последовательности в силу (3.2) определена $\xi^{\prime \prime}\left(\tau_{k}\right)$ и согласно (3.3) имеем

$$
0 \geqslant \xi^{\prime \prime}\left(\tau_{k}\right)=\left(\frac{1-\lambda}{2-\lambda}\right)^{\lambda} \xi\left(\tau_{k}\right)\left[\frac{1}{2-\lambda}\left(\frac{1-\lambda}{2-\lambda}\right)^{1-\lambda}-p\left(t_{k}\right) t_{k}^{1+\gamma}\left|\xi\left(\tau_{k}\right)\right|^{\sigma+\lambda-1}\right],
$$

где $t_{k}=e^{\tau_{k}}$. Следовательно,

$$
p\left(t_{k}\right) t_{k}^{1+\gamma}\left|\xi\left(\tau_{k}\right)\right|^{\sigma+\lambda-1} \geqslant \frac{1}{2-\lambda}\left(\frac{1-\lambda}{2-\lambda}\right)^{1-\lambda} \text { при } k=1,2, \ldots
$$

Учитывая теперь, что

$$
y\left(t_{k}\right)=t_{k}^{\frac{1-\lambda}{2-\lambda}} \xi\left(\tau_{k}\right), \quad y^{\prime}\left(t_{k}\right)=\frac{1-\lambda}{2-\lambda} t_{k}^{-\frac{1}{2-\lambda}} \xi\left(\tau_{k}\right)
$$

и принимая во внимание неравенства (3.1), (3.5), $\sigma+\lambda<1$ и $\sigma>-1$, из (2.1) при $s=t_{0}$ и $t=t_{k}$ получим

$$
\begin{aligned}
0 & <\rho_{1}(y)\left(t_{0}\right) \exp \left(-\int_{t_{0}}^{t_{k}} \frac{d p_{0}(\tau)}{p(\tau)}\right) \leqslant \rho_{1}(y)\left(t_{k}\right) \\
& =\frac{\left|\xi\left(\tau_{k}\right)\right|^{\sigma+1}}{t_{k} p\left(t_{k}\right)}\left[\left(\frac{1-\lambda}{2-\lambda}\right)^{2-\lambda}\left|\xi\left(\tau_{k}\right)\right|^{1-\sigma-\lambda}+\frac{2-\lambda}{\sigma+1} p\left(t_{k}\right) t_{k}^{1+\gamma}\right] \\
& \leqslant \frac{2-\lambda+(1-\lambda)(\sigma+1)}{\sigma+1} t_{k}^{\gamma}\left|\xi\left(\tau_{k}\right)\right|^{\sigma+1} \\
& \leqslant \frac{2-\lambda+(1-\lambda)(\sigma+1)}{\sigma+1}\left[\frac{1}{2-\lambda}\left(\frac{1-\lambda}{2-\lambda}\right)^{1-\lambda}\right]^{\frac{\sigma+1}{\sigma+\lambda-1}}\left[p\left(t_{k}\right) t_{k}^{2-\lambda}\right]^{\frac{\sigma+1}{1-\sigma-\lambda}} .
\end{aligned}
$$


Отсюда следует, что

$$
\begin{aligned}
& p\left(t_{k}\right) t_{k}^{2-\lambda} \exp \left(\frac{1-\sigma-\lambda}{\sigma+1} \int_{t_{0}}^{t_{k}} \frac{d p_{0}(\tau)}{p(\tau)}\right) \\
& \geqslant \frac{1}{2-\lambda}\left(\frac{1-\lambda}{2-\lambda}\right)^{1-\lambda}\left[\frac{(\sigma+1) \rho_{1}(y)\left(t_{0}\right)}{2-\lambda+(\sigma+1)(1-\lambda)}\right]^{\frac{1-\sigma-\lambda}{\sigma+1}}>0 \text { при } k=1,2, \ldots
\end{aligned}
$$

Однако при всех $k \in\{1,2, \ldots\}$ это неравенство не может соблюдаться, поскольку его левая часть в силу условий (1.3) и $\lim _{k \rightarrow+\infty} t_{k}=+\infty$ стремится к нулю при $k \rightarrow+\infty$. Полученное противоречие доказьвает, что наше предположение о существовании у уравнения (1.1) правильного колеблющегося решения было неверным. Значит, каждое правильное решение уравнения (1.1) является неколеблющимся. Теорема доказана.

ДОКАЗАТЕЛЬСТВО ТЕОРЕМЫ 2 проводится аналогично доказательству теоремы 1 с той лишь разницей что вместо (2.1) используются неравенства (2.2).

ДОКАЗАТЕЛЬСТВО ТЕОРЕМЫ 3. Предположим, что уравнение (1.1) имеет правильное колеблющееся решение $y$. Тогда это решение определено на некотором промежутке $\left[t_{0},+\infty\left[\left(t_{0} \geqslant b\right)\right.\right.$ и удовлетворяет неравенству $|y(t)|+\left|y^{\prime}(t)\right|>0$ при $t \geqslant t_{0}$. Следовательно, существуют последовательности $\left\{a_{k}\right\}$ и $\left\{b_{k}\right\}$, где $a_{1} \geqslant t_{0}$, такие, что

$$
\begin{gathered}
a_{k}<b_{k}<a_{k+1}, \quad \lim _{k \rightarrow+\infty} a_{k}=+\infty, \quad y\left(a_{k}\right)=y^{\prime}\left(b_{k}\right)=0 \\
\left.(-1)^{k} y(t)>0 \text { при } t \in\right] a_{k}, a_{k+1}[ \\
\left.(-1)^{k+1} y^{\prime}(t)>0 \text { при } t \in\right] b_{k}, b_{k+1}[, \quad k=1,2, \ldots
\end{gathered}
$$

Покажем сначала, что последовательность $\left\{a_{k}\left|y^{\prime}\left(a_{k}\right)\right|^{2-\lambda}\right\}$ является невозрастающей. Для этого, умножая (1.1) на $t\left|y^{\prime}(t)\right|^{1-\lambda} \operatorname{sign} y^{\prime}(t)$ и интегрируя на промежутке от $a_{k}$ до $a_{k+1}$, получим

$$
\begin{gathered}
\frac{a_{k+1}\left|y^{\prime}\left(a_{k+1}\right)\right|^{2-\lambda}}{2-\lambda}-\frac{a_{k}\left|y^{\prime}\left(a_{k}\right)\right|^{2-\lambda}}{2-\lambda}-\frac{1}{2-\lambda} \int_{a_{k}}^{a_{k+1}}\left|y^{\prime}(t)\right|^{2-\lambda} d t \\
=-\int_{a_{k}}^{a_{k+1}} t p(t)|y(t)|^{\sigma} y^{\prime}(t) \operatorname{sign} y(t) d t .
\end{gathered}
$$

А так как согласно лемме 2

$$
\int_{a_{k}}^{a_{k+1}}\left|y^{\prime}(t)\right|^{2-\lambda} d t=(1-\lambda) \int_{a_{k}}^{a_{k+1}} p(t)|y(t)|^{\sigma+1} d t
$$

то

$$
\begin{aligned}
& \frac{a_{k+1}\left|y^{\prime}\left(a_{k+1}\right)\right|^{2-\lambda}}{2-\lambda}-\frac{a_{k}\left|y^{\prime}\left(a_{k}\right)\right|^{2-\lambda}}{2-\lambda} \\
& \quad=\frac{1-\lambda}{2-\lambda} \int_{a_{k}}^{a_{k+1}} p(t)|y(t)|^{\sigma+1} d t-\int_{a_{k}}^{a_{k+1}} t p(t)|y(t)|^{\sigma} y^{\prime}(t) \operatorname{sign} y(t) d t .
\end{aligned}
$$


Отсюда следует, что

$$
\frac{a_{k+1}\left|y^{\prime}\left(a_{k+1}\right)\right|^{2-\lambda}}{2-\lambda}-\frac{a_{k}\left|y^{\prime}\left(a_{k}\right)\right|^{2-\lambda}}{2-\lambda}=-\frac{1}{\sigma+1} \int_{a_{k}}^{a_{k+1}} p(t) t^{1+\gamma}\left(\frac{|y(t)|^{\sigma+1}}{t^{\gamma}}\right)^{\prime} d t
$$

где $\gamma$ из (3.4).

В силу условий теоремы функция $p(t) t^{1+\gamma}$ не возрастает на промежутке $\left[t_{0},+\infty[\right.$. Поэтому, применяя к интегралу, стоящему в последнем соотношении справа, вторую теорему о среднем значении, получим

$$
\frac{a_{k+1}\left|y^{\prime}\left(a_{k+1}\right)\right|^{2-\lambda}}{2-\lambda}-\frac{a_{k}\left|y^{\prime}\left(a_{k}\right)\right|^{2-\lambda}}{2-\lambda}=-\frac{1}{\sigma+1} p\left(a_{k}\right) a_{k}^{1+\gamma} \xi_{k}^{-\gamma}\left|y\left(\xi_{k}\right)\right|^{\sigma+1},
$$

где $\xi_{k} \in\left[a_{k}, a_{k+1}\right]$.

Следовательно, при любом $k \in\{1,2, \ldots\}$

$$
a_{k+1}\left|y^{\prime}\left(a_{k+1}\right)\right|^{2-\lambda} \leqslant a_{k}\left|y^{\prime}\left(a_{k}\right)\right|^{2-\lambda}
$$

т.е. последовательность $\left\{a_{k}\left|y^{\prime}\left(a_{k}\right)\right|^{2-\lambda}\right\}$ является невозрастающей.

Ввиду установленного факта для некоторого числа $A>0$ соблюдаются неравенства

$$
a_{k}\left|y^{\prime}\left(a_{k}\right)\right|^{2-\lambda} \leqslant A, \quad k=1,2, \ldots
$$

Далее, покажем, что

$$
\int_{a_{2 k}}^{b_{2 k}} \frac{\left|y^{\prime}(t)\right|^{1-\lambda} y(t)}{t} d t \leqslant A \ln a_{2 k}, \quad k=1,2, \ldots
$$

Согласно (3.6) для любого $k \in\{1,2, \ldots\}$

$$
y\left(a_{2 k}\right)=y^{\prime}\left(b_{2 k}\right)=0
$$

и

$$
\left.y(t)>0, \quad y^{\prime}(t)>0 \text { при } t \in\right] a_{2 k}, b_{2 k}[.
$$

Поэтому, умножая (1.1) на $\left|y^{\prime}(t)\right|^{1-\lambda}(t \ln t)$ и интегрируя на промежутке от $a_{2 k}$ до $b_{2 k}$, получим

$$
\begin{gathered}
-\frac{1}{2-\lambda} \int_{a_{2 k}}^{b_{2 k}}(1+\ln t)\left|y^{\prime}(t)\right|^{2-\lambda} d t+\int_{a_{2 k}}^{b_{2 k}}(t \ln t) p(t)|y(t)|^{\sigma} y^{\prime}(t) d t \\
=a_{2 k} \ln a_{2 k} \frac{\left|y^{\prime}\left(a_{2 k}\right)\right|^{2-\lambda}}{2-\lambda}, \quad k=1,2, \ldots
\end{gathered}
$$

Принимая во внимание лемму 2 , запишем эти соотношения в виде

$$
\begin{aligned}
& -\frac{1-\lambda}{2-\lambda} \int_{a_{2 k}}^{b_{2 k}}(1+\ln t) p(t)|y(t)|^{\sigma+1} d t+\frac{1}{2-\lambda} \int_{a_{2 k}}^{b_{2 k}} \frac{\left|y^{\prime}(t)\right|^{1-\lambda} y(t)}{t} d t \\
& \quad+\int_{a_{2 k}}^{b_{2 k}}(t \ln t) p(t)|y(t)|^{\sigma} y^{\prime}(t) d t=a_{2 k} \ln a_{2 k} \frac{\left|y^{\prime}\left(a_{2 k}\right)\right|^{2-\lambda}}{2-\lambda}, \quad k=1,2, \ldots
\end{aligned}
$$


Отсюда, учитывая, что

$$
\begin{gathered}
\int_{a_{2 k}}^{b_{2 k}}(t \ln t) p(t)|y(t)|^{\sigma} y^{\prime}(t) d t-\frac{1-\lambda}{2-\lambda} \int_{a_{2 k}}^{b_{2 k}}(1+\ln t) p(t)|y(t)|^{\sigma+1} d t \\
=\frac{1}{\sigma+1} \int_{a_{2 k}}^{b_{2 k}} p(t)(t \ln t)^{1+\gamma}\left(\frac{|y(t)|^{\sigma+1}}{(t \ln t)^{\gamma}}\right)^{\prime} d t, \quad k=1,2, \ldots
\end{gathered}
$$

где $\gamma$ из (3.4), находим

$$
\begin{aligned}
& \frac{1}{2-\lambda} \int_{a_{2 k}}^{b_{2 k}} \frac{\left|y^{\prime}(t)\right|^{1-\lambda} y(t)}{t} d t+\frac{1}{\sigma+1} \int_{a_{2 k}}^{b_{2 k}} p(t)(t \ln t)^{1+\gamma}\left(\frac{|y(t)|^{\sigma+1}}{(t \ln t)^{\gamma}}\right)^{\prime} d t \\
& \quad=a_{2 k} \ln a_{2 k} \frac{\left|y^{\prime}\left(a_{2 k}\right)\right|^{2-\lambda}}{2-\lambda}, \quad k=1,2, \ldots
\end{aligned}
$$

Поскольку функция $p(t)(t \ln t)^{1+\gamma}$ в силу условий теоремы не возрастает на промежутке $\left[t_{0},+\infty[\right.$, согласно второй теореме о среднем значении

$$
\int_{a_{2 k}}^{b_{2 k}} p(t)(t \ln t)^{1+\gamma}\left(\frac{|y(t)|^{\sigma+1}}{(t \ln t)^{\gamma}}\right)^{\prime} d t=p\left(a_{2 k}\right)\left(a_{2 k} \ln a_{2 k}\right)^{1+\gamma} \frac{\left|y\left(\xi_{k}\right)\right|^{\sigma+1}}{\left(\xi_{k} \ln \xi_{k}\right)^{\gamma}} \geqslant 0
$$

где $\xi_{k} \in\left[a_{2 k}, b_{2 k}\right]$.

Поэтому из (3.10) с учетом (3.7) имеем

$$
\int_{a_{2 k}}^{b_{2 k}} \frac{\left|y^{\prime}(t)\right|^{1-\lambda} y(t)}{t} d t \leqslant a_{2 k} \ln a_{2 k}\left|y^{\prime}\left(a_{2 k}\right)\right|^{2-\lambda} \leqslant A \ln a_{2 k}, \quad k=1,2, \ldots
$$

Используя теперь оценки (3.7) и (3.8), докажем, что

$$
\int_{a_{2 k}}^{b_{2 k}}\left|y^{\prime}(t)\right|^{2-\lambda} d t \leqslant A\left(1+2 \ln a_{2 k}\right), \quad k=1,2, \ldots
$$

Так как в силу (1.1) и (3.9) для любого $k \in\{1,2, \ldots\}$

$$
\left(\left(t-a_{2 k}\right) y^{\prime}(t)-y(t)\right)^{\prime}=-p(t)\left(t-a_{2 k}\right)|y(t)|^{\sigma}\left|y^{\prime}(t)\right|^{\lambda}<0
$$

при $t \in] a_{2 k}, b_{2 k}\left[\right.$, а $y\left(a_{2 k}\right)=0$, то

$$
0 \leqslant\left(t-a_{2 k}\right) y^{\prime}(t) \leqslant y(t) \quad \text { при } \quad t \in\left[a_{2 k}, b_{2 k}\right] .
$$

Отсюда следует, что для любого $k \in\{1,2, \ldots\}$

$$
\begin{aligned}
\int_{a_{2 k}}^{b_{2 k}}\left|y^{\prime}(t)\right|^{2-\lambda} d t & =\int_{a_{2 k}}^{b_{2 k}} \frac{\left(t-a_{2 k}\right)+a_{2 k}}{t}\left|y^{\prime}(t)\right|^{2-\lambda} d t \\
& \leqslant \int_{a_{2 k}}^{b_{2 k}} \frac{\left|y^{\prime}(t)\right|^{1-\lambda} y(t)}{t} d t+a_{2 k} \int_{a_{2 k}}^{b_{2 k}} \frac{\left|y^{\prime}(t)\right|^{2-\lambda}}{t} d t
\end{aligned}
$$




$$
\begin{aligned}
\int_{a_{2 k}}^{b_{2 k}} \frac{\left|y^{\prime}(t)\right|^{2-\lambda}}{t} d t & =\int_{a_{2 k}}^{b_{2 k}} \frac{\left(t-a_{2 k}\right)+a_{2 k}}{t^{2}}\left|y^{\prime}(t)\right|^{2-\lambda} d t \\
& \leqslant \int_{a_{2 k}}^{b_{2 k}} \frac{\left|y^{\prime}(t)\right|^{1-\lambda} y(t)}{t^{2}} d t+a_{2 k} \int_{a_{2 k}}^{b_{2 k}} \frac{\left|y^{\prime}(t)\right|^{2-\lambda}}{t^{2}} d t .
\end{aligned}
$$

Из этих неравенств, учитывая, что $y^{\prime}(t) \leqslant y^{\prime}\left(a_{2 k}\right)$ при $t \in\left[a_{2 k}, b_{2 k}\right]$ и используя неравенства (3.7), (3.8), находим

$$
\begin{aligned}
\int_{a_{2 k}}^{b_{2 k}}\left|y^{\prime}(t)\right|^{2-\lambda} d t \leqslant \int_{a_{2 k}}^{b_{2 k}} \frac{\left|y^{\prime}(t)\right|^{1-\lambda} y(t)}{t} d t+a_{2 k} \int_{a_{2 k}}^{b_{2 k}} \frac{\left|y^{\prime}(t)\right|^{1-\lambda} y(t)}{t^{2}} d t \\
\quad+a_{2 k}^{2}\left|y^{\prime}\left(a_{2 k}\right)\right|^{2-\lambda} \int_{a_{2 k}}^{b_{2 k}} \frac{d t}{t^{2}} \leqslant 2 \int_{a_{2 k}}^{b_{2 k}} \frac{\left|y^{\prime}(t)\right|^{1-\lambda} y(t)}{t} d t+a_{2 k}\left|y^{\prime}\left(a_{2 k}\right)\right|^{2-\lambda} \\
\leqslant 2 A \ln a_{2 k}+A=A\left(1+2 \ln a_{2 k}\right), \quad k=1,2, \ldots
\end{aligned}
$$

Установленная оценка (3.11) позволяет теперь легко завершить доказательство теоремы.

В силу условий (3.9) и неравенства Гёльдера

$$
\begin{aligned}
|y(t)| & =\int_{a_{2 k}}^{t}\left|y^{\prime}(\tau)\right| d \tau \leqslant\left(t-a_{2 k}\right)^{\frac{1-\lambda}{2-\lambda}}\left(\int_{a_{2 k}}^{t}\left|y^{\prime}(\tau)\right|^{2-\lambda} d \tau\right)^{\frac{1}{2-\lambda}} \\
& \leqslant t^{\frac{1-\lambda}{2-\lambda}}\left(\int_{a_{2 k}}^{b_{2 k}}\left|y^{\prime}(t)\right|^{2-\lambda} d t\right)^{\frac{1}{2-\lambda}} \text { при } t \in\left[a_{2 k}, b_{2 k}\right], \quad k=1,2, \ldots
\end{aligned}
$$

Поэтому, принимая во внимание лемму 2 , получим

$$
\begin{aligned}
& \int_{a_{2 k}}^{b_{2 k}} \frac{\left|y^{\prime}(t)\right|^{2-\lambda}}{1-\lambda} d t=\int_{a_{2 k}}^{b_{2 k}} p(t)|y(t)|^{\sigma+1} d t \\
& \quad \leqslant\left(\int_{a_{2 k}}^{b_{2 k}}\left|y^{\prime}(t)\right|^{2-\lambda} d t\right)^{\frac{\sigma+1}{2-\lambda}} \int_{a_{2 k}}^{b_{2 k}} p(t) t^{\frac{(\sigma+1)(1-\lambda)}{2-\lambda}} d t, \quad k=1,2, \ldots
\end{aligned}
$$

А так как для некоторого $\varepsilon>0$, удовлетворяющего неравенству (1.5), функция (1.6) не возрастает на промежутке $\left[t_{0},+\infty[\right.$, будем иметь

$$
\begin{aligned}
\int_{a_{2 k}}^{b_{2 k}} & \frac{\left|y^{\prime}(t)\right|^{2-\lambda}}{1-\lambda} d t \leqslant C\left(\int_{a_{2 k}}^{b_{2 k}}\left|y^{\prime}(t)\right|^{2-\lambda} d t\right)^{\frac{\sigma+1}{2-\lambda}} \int_{a_{2 k}}^{+\infty} \frac{d t}{t(\ln t)^{\frac{\sigma+1}{2-\lambda}+\varepsilon}} \\
= & \frac{(2-\lambda) C}{\sigma+\lambda-1+\varepsilon(2-\lambda)}\left(\int_{a_{2 k}}^{b_{2 k}}\left|y^{\prime}(t)\right|^{2-\lambda} d t\right)^{\frac{\sigma+\lambda-1}{2-\lambda}}\left(\ln a_{2 k}\right)^{\frac{1-\sigma-\lambda}{2-\lambda}-\varepsilon}, \quad k=1,2, \ldots,
\end{aligned}
$$


где $C$ - положительная постоянная. Отсюда с учетом оценки (3.11) находим

$$
\begin{aligned}
0 & <\frac{1}{1-\lambda} \leqslant \frac{(2-\lambda) C}{\sigma+\lambda-1}\left(\int_{a_{2 k}}^{b_{2 k}}\left|y^{\prime}(t)\right|^{2-\lambda} d t\right)^{\frac{\sigma+\lambda-1}{2-\lambda}}\left(\ln a_{2 k}\right)^{\frac{1-\sigma-\lambda}{2-\lambda}-\varepsilon} \\
& \leqslant \frac{(2-\lambda) C A^{\frac{\sigma+\lambda-1}{2-\lambda}}}{\sigma+\lambda-1+\varepsilon(2-\lambda)}\left(1+2 \ln a_{2 k}\right)^{\frac{\sigma+\lambda-1}{2-\lambda}}\left(\ln a_{2 k}\right)^{\frac{1-\sigma-\lambda}{2-\lambda}-\varepsilon}, \quad k=1,2, \ldots
\end{aligned}
$$

Однако, при всех $k \in\{1,2, \ldots\}$ это неравенство вьполняться не может, поскольку его правая часть в силу условия $\lim _{k \rightarrow+\infty} a_{k}=+\infty$ стремится к нулю при $k \rightarrow+\infty$.

Полученное противоречие доказывает, что наше предположение о существовании у уравнения (1.1) правильного колеблюшегося решения было неверным. Следовательно, каждое его правильное решение является неколеблющимся. Теорема полностью доказана.

\section{СПИСОК ЦИТИРОВАННОЙ ЛИТЕРАТУРЫ}

[1] Кигурадзе И.Т. Об условиях колеблемости решений уравнения $u^{\prime \prime}+a(t)|u|^{n} \operatorname{sign} u=0$ // Cas. Pest. Mat. 1962. V. 87. № 4. P. 492-495.

[2] Nehari Z. A nonlinear oscillation problem // J. Different. Equat. 1969. V. 5. № 3. P. 452-460.

[3] Kuo-Liang Chion. A second order nonlinear oscillation theorem // SIAM J. Appl. Math. 1971. V. 21. № 2. P. 221-224.

[4] Kuo-Liang Chion. A nonoscillation theorem for the superlinear case of second-order differential equations // SIAM J. Appl. Math. 1972. V. 23. № 4. P. 456-459.

[5] Wong J.S. W. Remarks on nonoscillation theorem for a second-order nonlinear differential equation // Proc. Amer. Math. Soc. 1981. V. 83. № 3. P. 541-546.

[6] Кигурадзе И.Т., Чантурия Т. А. Асимптотические свойства решений неавтономных обыкновенных дифференциальных уравнений. М.: Наука, 1990.

[7] Kiguradze I. T. On the oscillatory and monotone solution of ordinary differential equation // Arch. Mat. 1, Scripta Fac. Sci. Nat. 1978. V. 14. P. 21-44.

[8] Wong J.S. W. On the generalized Emden-Fowler equation // SIAM Rev. 1975. V. 17. № 2. P. $339-360$.

[9] Схаляхо Ч. А. О неколеблемости решений одной системы двух дифференциальных уравнений // Cas. Pest. Mat. 1982. V. 107. P. 139-142.

[10] Евтухов В. М. Об условиях колеблемости и неколеблемости решений одного полулинейного дифференциального уравнения второго порядка // Укр. матем. ж. 1994. Т. 46. № 7. С. 833-841.

[11] Мирзов Д. Д. О неколеблемости решений одной системы дифференциальных уравнений // Матем. заметки. 1978. Т. 23. № 3. С. 401-404.

[12] Mirzov J. D. On some analogs of Sturm's and Kueser's theorems for nonlinear systems // J. Math. Anal and Appl. 1976. V. 53. № 1. P. 418-425.

Одесский государственный университет им. И.И. Мечникова

Поступило

E-mail: evt@evtukhov.imem.odessa.ua

16.04.1997

Исправленный вариант

26.07.1999 